\title{
Partial and total thermal neutron capture cross sections for non-destructive assay and transmutation monitoring of ${ }^{99} \mathrm{Tc}$
}

\author{
By G. L. Molnár ${ }^{1, *}$, T. Belgya ${ }^{1}$, Zs. Révay ${ }^{1}$ and S. M. Qaim ${ }^{2}$ \\ ${ }^{1}$ Institute of Isotope and Surface Chemistry, Chemical Research Centre, P.O. Box 77, H-1525 Budapest, Hungary \\ 2 Institut für Nuklearchemie, Forschungszentrum Jülich GmbH, Postfach 1913, D-52425 Jülich, Germany
}

(Received May 27, 2002; accepted July 22, 2002)

\begin{abstract}
Thermal neutron capture / Gamma rays / Cold neutrons / Cross section / $k_{0}$ method / PGAA / NAA / Technetium 99 / Technetium 100 / Beta decay
\end{abstract}

Summary. Accurate partial gamma-ray production cross sections were determined for the prompt and radioactive product decay gamma rays following cold neutron capture in ${ }^{99} \mathrm{Tc}$. They can be used for non-destructive assay of technetium by prompt gamma activation analysis (PGAA) and neutron activation analysis (NAA), offering orders of magnitude higher analytical sensitivities than passive gamma-ray counting. A lower limit of $21.21 \pm 0.17 \mathrm{~b}$ was also deduced for the thermal-neutron-capture cross section.

\section{Introduction}

The artificial radionuclide ${ }^{99} \mathrm{Tc}$ is important from many viewpoints. Its $6 \mathrm{~h}$ isomer, ${ }^{99 \mathrm{~m}} \mathrm{Tc}$, is the most frequently used radioisotope in diagnostic nuclear medicine. The isomer decays solely to the ground state by emitting a single $142 \mathrm{keV} \gamma$-ray, which is very suitable for Single Photon Emitted Computed Tomography (SPECT). Both forms are produced in nuclear fission, partly independently but mostly via the beta decay of ${ }^{99} \mathrm{Mo}$. Along with ${ }^{129} \mathrm{I},{ }^{99} \mathrm{Tc}$ constitutes 95 percent of the long-lived (> 500 a) nuclear waste from pressurised water moderated reactors. Its ability to migrate and the efficient intake by living organisms make ${ }^{99} \mathrm{Tc}$ a hazardous radiotoxic material. Furthermore, the extremely long half-life $\left(2.1 \times 10^{5}\right.$ a), the low beta-decay endpoint energy $(294 \mathrm{keV})$, and the single weak $\left(6.5 \times 10^{-4} \%\right)$ low-energy $(89.5 \mathrm{keV}) \gamma$-ray [1], superimposed on a high bremsstrahlung background, make the non-destructive assay of ${ }^{99} \mathrm{Tc}$ with passive nuclear methods difficult.

Fortunately, ${ }^{99} \mathrm{Tc}$ can be transformed by neutron capture into ${ }^{100} \mathrm{Tc}$, which decays with a $15 \mathrm{~s}$ half-life to stable ${ }^{100} \mathrm{Ru}$. This is the basis of transmutation of technetium contained in the nuclear waste [2]. The $\gamma$-radiations produced can in principle be used for assaying technetium either by prompt

\footnotetext{
*Author for correspondence (E-mail: molnar@iki.kfki.hu).
}

gamma activation analysis (PGAA) or by conventional neutron activation analysis (NAA), depending on the type of radiation utilised. The NAA technique has been applied in the TARC experiment at CERN [2] for controlling the transmutation process. In the present work, accurate partial and total capture cross sections have been determined for the major $\gamma$-rays from the ${ }^{99} \mathrm{Tc}(n, \gamma){ }^{100} \mathrm{Tc}$ capture reaction and the subsequent beta decay of $15 \mathrm{~s}{ }^{100} \mathrm{Tc}$ at the cold neutron PGAA facility of the Budapest Research Reactor.

\section{Experimental procedures}

The number of counts for a particular neutron capture $\gamma$-ray is proportional to the amount of target material and the measurement time. The count rate is governed by the partial $\gamma$-ray production cross section

$$
\sigma_{\gamma}=P_{\gamma} \sigma
$$

where $\sigma$ is the total capture cross section and $P_{\gamma}$ is the emission probability per capture or per beta decay for a particular $\gamma$-ray. The partial cross section, once measured, can be used to determine the amount of material. It is equivalent to the prompt $k_{0}$ factor, which is practically the partial cross section per atomic mass, related to the similar quantity for a selected comparator element [3]. In addition, $\sigma_{\gamma}$ can also be used to infer the total capture cross section if $P_{\gamma}$ values are available.

The partial cross sections were determined for the strongest $\gamma$-rays by using an internal standard method based on stoichiometric compounds [3]. The partial cross sections of analyte, $x$, and comparator, $c$, are related as

$$
\frac{\sigma_{\gamma, x}}{\sigma_{\gamma, c}}=\frac{I_{\gamma, x}}{I_{\gamma, c}} \frac{n_{c}}{n_{x}}
$$

where $I_{\gamma}$ is the $\gamma$-ray yield and $n$ is the stoichiometric number. Note that this ratio is just the $k_{0}$ factor defined by Eq. (6) in Ref. [3]

$$
k_{0, c}=\frac{I_{\gamma, x}}{I_{\gamma, c}} \frac{n_{c} M_{c}}{n_{x} M_{x}}
$$


except for the normalisation of $\gamma$-ray yields to the respective atomic mass $M$. These simple relationships are valid only for thin targets, and nuclides for which the cross section obeys the $1 / v$ law.

Ammonium pertechnetate, $\mathrm{NH}_{4} \mathrm{TcO}_{4}$, was chosen due to its chemical stability. Hydrogen, emitting a single $2223 \mathrm{keV}$ $\gamma$-ray is an ideal comparator, and its total capture cross section $(0.3326 \pm 0.0007 \mathrm{~b})$ is also accurately known [4]. An amount of $0.1135 \mathrm{~g}$ ammonium pertechnetate powder (supplied by Oak Ridge National Laboratory, USA) was sealed in a thin Teflon bag and placed in the guided cold neutron beam with a thermal-equivalent flux of $5 \times 10^{7} \mathrm{~cm}^{-2} \mathrm{~s}^{-1}$. The in-beam spectrum was collected with our standard Compton-suppressed HPGe detector (25\% efficiency) [5], placed at $23.5 \mathrm{~cm}$ from the target to keep the count rate sufficiently low and suppress coincidence summing. The relative efficiency was determined using radioactive sources and capture $\gamma$-rays as described elsewhere [6]. Partial cross-sections for the strong 172, 223 and $263 \mathrm{keV}$ capture $\gamma$-rays $[7,8]$ as well as for the 539 and $591 \mathrm{keV}$ $\gamma$-rays from ${ }^{100} \mathrm{Tc}$ decay [9] were determined from the same in-beam spectrum. In the latter case, however, the yield of a decay line is proportional to the effective counting time:

$$
t \rightarrow t\left(1-\frac{1-\exp (-\lambda t)}{\lambda t}\right) .
$$

Here $\lambda$ is the decay constant. This correction becomes negligible after about thousand half-lives. The spectrum shown in Fig. 1 was collected for about 18.5 hours to attain the highest possible statistics. Self-absorption in the target amounted to a few percent at the lowest energies, and was accounted for by calculation using the PC code XMuDat [10].

In order to unveil the possible interferences, the prompt (capture) and decay $\gamma$-rays were studied in greater detail by using a target of metallic ${ }^{99} \mathrm{Tc}$ (supplied by Oak Ridge National Laboratory, USA). An amount of $0.5 \mathrm{~g}$ was pressed to a disc of $13 \mathrm{~mm}$ diameter and encapsulated in an aluminium container with $0.2 \mathrm{~mm}$ wall thickness. Applying a beam chopper with about $25 \mathrm{~Hz}$ repetition rate and $50 \%$ duty cycle, the in-beam spectrum (containing prompt and decay $\gamma$-lines together) and the off-beam decay spectrum could be collected simultaneously. A background spectrum was also measured under similar conditions. No contaminants were found to disturb the $\gamma$-rays of interest, nor did the major prompt

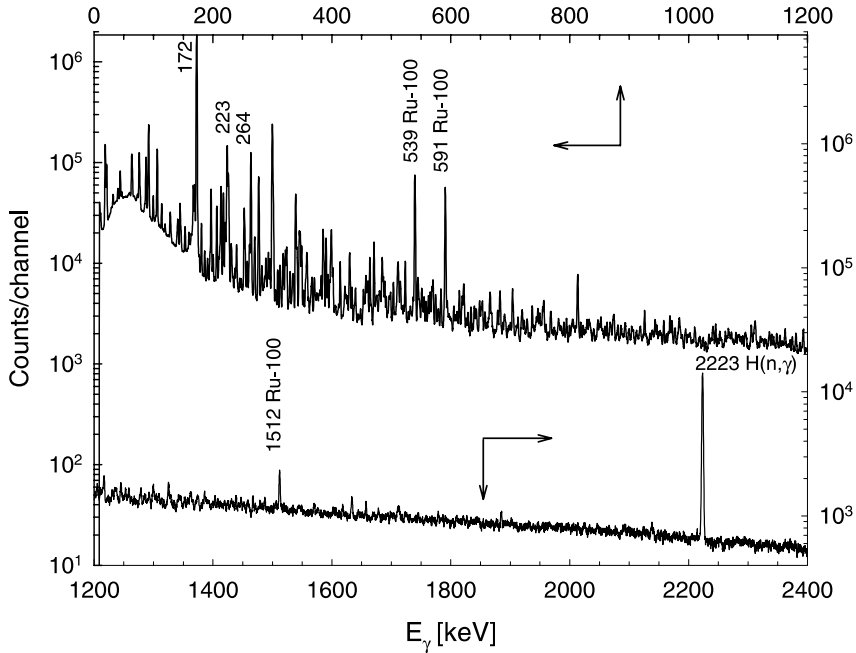

Fig. 1. Gamma-ray spectrum from the ${ }^{99} \mathrm{Tc}(n, \gamma)$ reaction using ammonium pertechnetate target. The ${ }^{100} \mathrm{Tc}$ decay lines are labelled by the excited product nucleus ${ }^{100} \mathrm{Ru}$.

and decay lines interfere. However, in the measurements with ammonium pertechnetate the correction for hydrogen background due to air moisture $(0.028 \mathrm{cps})$ amounted to $1 \%$.

Finally, the spontaneous decay of ${ }^{99} \mathrm{Tc}$ was also measured, using an unshielded smaller (15\% efficiency) HPGe detector with a thin carbon fibre window at a similar targetto-detector distance. The count rate for the $89.5 \mathrm{keV} \gamma$-ray, adjusted for the standard spectrometer, served as the basis of comparison of the analytical sensitivities.

\section{Results and discussion}

The thermal partial cross sections for the strongest $\gamma$-rays have been determined from the $\gamma$-ray yields measured in cold neutron capture and subsequent beta decay, respectively, according to Eq. (2). A $1 / v$ relationship was assumed to hold also for ${ }^{99} \mathrm{Tc}$, and the standard value of $0.3326 \pm 0.0007 \mathrm{~b}$ [4] was used for the thermal neutron capture cross section of the hydrogen comparator. The new cross sections are listed in Table 1. The uncertainty is about $0.9 \%$, which essentially comes from the relative efficiency calibration. This should be contrasted with the $9 \%$ uncertainty of the emission probability for the $539 \mathrm{keV}$ decay $\gamma$-ray, determined in [9],
Table 1. Partial $\gamma$-ray production cross sections of capture and decay lines for a ${ }^{99} \mathrm{Tc}$ target.

\begin{tabular}{rcccc}
\hline $\begin{array}{l}E_{\gamma} \\
(\mathrm{keV})\end{array}$ & Origin & $\begin{array}{c}P_{\gamma} \\
(\gamma / 100 \text { captures } \\
\text { or decays })\end{array}$ & $\begin{array}{c}\sigma_{\gamma}{ }^{a} \\
(\mathrm{~b})\end{array}$ & $\begin{array}{c}\text { Sensitivity } \\
(\mathrm{cps} / \mathrm{mg})\end{array}$ \\
\hline 172.1 & ${ }^{99} \mathrm{Tc}(n, \gamma)^{100} \mathrm{Tc}$ & $67 \pm 6$ & $16.61 \pm 0.15$ & 3.0 \\
223.4 & ${ }^{99} \mathrm{Tc}(n, \gamma)^{100} \mathrm{Tc}$ & $6.1 \pm 0.6$ & $1.472 \pm 0.013$ & 0.24 \\
263.5 & ${ }^{99} \mathrm{Tc}(n, \gamma)^{100} \mathrm{Tc}$ & $5.9 \pm 0.5$ & $1.425 \pm 0.012$ & 0.21 \\
539.5 & ${ }^{100} \mathrm{Tc}\left(\beta^{-}\right)^{100} \mathrm{Ru}$ & $6.6 \pm 0.6^{b}$ & $1.604 \pm 0.014$ & 0.14 \\
590.7 & ${ }^{100} \mathrm{Tc}\left(\beta^{-}\right)^{100} \mathrm{Ru}$ & $5.5 \pm 0.4$ & $1.296 \pm 0.011$ & 0.10 \\
89.5 & ${ }^{99} \mathrm{Tc}\left(\beta^{-}\right)^{100} \mathrm{Ru}$ & $(6.5 \pm 1.5) \times 10^{-4 c}$ & & $4.3 \times 10^{-3}$ \\
\hline
\end{tabular}

a: To obtain $k_{0}\left({ }^{1} \mathrm{H}\right)$ multiply by $0.0304(3)$, to obtain $k_{0}\left({ }^{198} \mathrm{Au}\right)$ multiply by $0.687(6)$; b: Value from Ref. [9], used for normalisation here;

c: From Ref. [1]. 
Table 2. Inferred total thermal-neutroncapture cross section of ${ }^{99} \mathrm{Tc}$.

\begin{tabular}{lccl}
\hline Method & Basis & $\begin{array}{c}\sigma \\
(\mathrm{b})\end{array}$ & Comment \\
\hline${ }^{100} \mathrm{Tc}\left(\beta^{-}\right){ }^{100} \mathrm{Ru}$ & $539 \gamma$ & $24.7 \pm 2.3$ & with $P_{\gamma}$ from Ref. [9] \\
& $591 \gamma$ & $23.9 \pm 1.8$ & \\
& Average & $24.3 \pm 2.2$ & unweighted average \\
${ }^{99} \mathrm{Tc}(n, \gamma){ }^{100} \mathrm{Tc}$ & $\sum \sigma_{\gamma}$ g.s. & $21.21 \pm 0.17$ & lower limit \\
& & $26.5 \pm 2.6$ & missing $\sim 20 \%$ added \\
\hline
\end{tabular}

which was used in Table 1 for renormalising the measured $\gamma$-ray yields. The reported uncertainty for absolute intensity of the ${ }^{99} \mathrm{Tc}$ decay $\gamma$-ray is even higher, about $23 \%$ [1].

Table 1 clearly demonstrates the order-of-magnitude differences in partial cross sections and $\gamma$-ray intensities. For ${ }^{99} \mathrm{Tc}$ assay, the highest sensitivity is offered by PGAA based on the detection of the $172 \mathrm{keV}$ line, then follows NAA, while the least favourable method is the passive $\gamma$-ray counting. For orientation, analytical sensitivities characteristic of our measuring system have also been included in Table 1, in units of counts per second per milligram of material. It is striking that the sensitivity for the $172 \mathrm{keV}$ capture line is thousand times higher than for the $89.5 \mathrm{keV}$ line from spontaneous decay of ${ }^{99} \mathrm{Tc}$. On the other hand, the corresponding total rate is only doubled, i.e. $18 \mathrm{cps}$ and $9.6 \mathrm{cps}$, respectively, for the energy range of $7 \mathrm{keV}$ to $4 \mathrm{MeV}$.

The precisely measured partial cross sections can also be used for determining the total capture cross section of ${ }^{99} \mathrm{Tc}$ according to Eq. (1). The $P_{\gamma}$ values of the 539 and $591 \mathrm{keV}$ transitions were recently redetermined as $(6.6 \pm 0.6) \%$ and $(5.5 \pm 0.4) \%$, respectively (Table 3 of Ref. [9]. Note that the uncertainties have been slightly reduced in the text and abstract!) The obtained values and their unweighted average of $24.3 \pm 2.2 \mathrm{~b}$ (with the $9 \%$ systematic uncertainty kept) are listed in Table 2.

The capture cross section can also be determined in an independent way, by using the neutron capture $\gamma$-rays. The sum of $\sigma_{\gamma}$ values for all ground state transitions, when each one is corrected for internal conversion, $\alpha$, gives the total capture cross section:

$$
\sigma=\sum_{\text {g.s. }} \sigma_{\gamma}(1+\alpha) .
$$

The firmly established 172, 223, 264, 341 and $640 \mathrm{keV}$ ground state transitions [7,8] give a lower limit of $21.21 \pm 0.17 \mathrm{~b}$. Since the sum of the $P_{\gamma}$ values, obtained by normalising to the $591 \mathrm{keV}$ decay line [9], amounts to only $80 \%$, a correction has been made for the missing $20 \%$ carried by the unidentified ground-state transitions. We obtain $26.5 \pm 2.6 \mathrm{~b}$, as shown in Table 2 , where the above mentioned $9 \%$ systematic uncertainty of the decay data is added quadratically to the total cross section uncertainty. The uncertainty of the $20 \%$ correction is difficult to estimate, hence it has been ignored in the error calculations.

According to the EXFOR database [11], the literature values for the ${ }^{99} \mathrm{Tc}$ capture cross section range from $19 \pm 2 \mathrm{~b}$ to $24 \pm 4 \mathrm{~b}$. The most recent and accurate value is $22.9 \pm 2.6 \mathrm{~b}$, obtained by Harada et al. [12] using the activation method. (Note that the systematic uncertainty is suppressed in their Abstract). While their value agrees with both cross section values from the present work, all are tied up with the nearly $10 \%$ uncertainty of the decay $\gamma$-ray intensity. Hence, it can only be concluded that the new number of $21.21 \pm 0.17 \mathrm{~b}$ is a firm lower limit to the capture cross section, and the actual value should be higher.

In conclusion, the new partial $\gamma$-ray production cross sections for neutron capture and decay $\gamma$-rays allow for non-destructive assay of the ${ }^{99} \mathrm{Tc}$ long-lived fission product and for on-line monitoring its transmutation rate into stable ${ }^{100} \mathrm{Ru}$ by using NAA or PGAA. Besides the much better accuracy, the analytical sensitivities are about fifty times higher for NAA and thousand times higher for PGAA, in comparison to passive $\gamma$-ray counting. Naturally, the sensitivity of NAA is orders of magnitude higher when the irradiation takes place in a reactor, instead of a neutron beam, due to the much higher neutron flux.

Acknowledgment. This work was partly supported by the EU5 Framework Programme under contract HPRI-CT-1999-00099. Thanks are also due to the Budapest Reactor staff for the excellent working conditions.

\section{References}

1. Peker, L. K.: Nucl. Data Sheets 73, 1 (1994).

2. Abánades, A., Aleixandre, J., Andriamonje, S., Angelopoulos, A., Apostolakis, A., Arnould, H., Belle, E., Bompas, C. A., Brozzi, D., Bueno, J., Buono, S., Carminati, F., Casagrande, F., Cennini, P., Collar, J. I., Cerro, E., Del Moral, R., Diez, S., Dumps, L., Eleftheriadis, C., Embid, M., Fernández, R., Galvez, J., Garcia, J., Geles, C., Giorni, A., Gonzalez, E., Gonzalez, O., Goulas, I., Heuer, D., Hussonnois, M., Kadi, Y., Karaiskos, P., Kitis, G., Klapisch, R., Kokkas, P., Lacoste, V., Le Naour, C., Lopez, C., Loiseaux, J. M., Martinez-Val, J. M., Meplan, O., Nifenecker, H., Oropesa, J., Papadopoulos, I., Pavlopoulos, P., Perez-Enciso, E., Perez-Navarro, A., Perlado, M., Placci, A., Poza, M., Revol, J. P., Rubbia, C., Rubio, J. A., Sakelliou, L., Saldana, F., Savvidis, E., Schussler, F., Sirvent, C., Tamarit, J., Trubert, D., Tzima, A., Viano, J. B., Vieira, S., Vlachoudis, V., Zioutas, K.: Nucl. Instrum. Methods Phys. Res., Sect. A 478, 577 (2002).

3. Molnár, G. L., Révay, Z., Paul, R. L., Lindstrom, R. M.: J. Radioanal. Nucl. Chem. 234, 21 (1998).

4. Mughabghab, S. F., Divadeenam, M., Holden, N. E.: Neutron Cross Sections. Academic Press, New York (1981).

5. Molnár, G., Belgya, T., Dabolczi, L., Fazekas, B., Révay, Z., Veres, Á., Bikit, I., Kiss, Z., Östör, J.: J. Radioanal. Nucl. Chem. 215, 111 (1997).

6. Baglin, C. M., Browne, E., Norman, E. B., Molnár, G. L., Belgya, T., Révay, Z., Szelecsényi, F.: Nucl. Instrum. Methods Phys. Res., Sect. A 481, 365 (2001).

7. Pinston, J. A., Mampe, W., Roussille, R., Schreckenbach, K., Heck, D., Börner, H. G., Koch, H. R., Andre, S., Barneoud, D.: Nucl. Phys. A 321, 25 (1979).

8. Singh, B.: Nucl. Data Sheets 81, 1 (1997).

9. Furutaka, K., Nakamura, S., Harada, H., Katoh, T., Fujii, T., Yamana, H.: J. Nucl. Sci. Technol. 38, 1035 (2001). 
10. Nowotny, R.: XMuDat: Photon attenuation data on PC, Version 1.0.1 of August 1998. Report IAEA-NDS-195, International Atomic Energy Agency, Vienna (1998).

11. EXFOR Experimental Nuclear Reaction Data Library (in USA usually called CSISRS). This data base contains data compiled as the result of the cooperative efforts of the worldwide Nuclear Reaction Data Center Network, coordinated by the IAEA Nuclear Data Section (2002).

12. Harada, H., Nakamura, S., Katoh, T., Ogata, Y.: J. Nucl. Sci. Technol. 32, 395 (1995). 